\title{
EVALUACIÓN DE TIPOS DE SEMILLA Y ÉPOCA DE CORTE PARA LA SIEMBRA DE BATATA, Ipomoea batatas (L.) Lam, EN LA REGIÓN CARIBE DE COLOMBIA
}

\author{
Támara Morelos, R. e.1; García PeñA, J. A. ${ }^{2}$; \\ Espitia Montes, A. A. ${ }^{2}$; Regino Hernández, S. M. ${ }^{2}$; \\ Pérez Cantero, S. P. ${ }^{2}$ \& Contreras Santos, J. L. ${ }^{2}$
}

\begin{abstract}
RESUMEN
El objetivo fue evaluar diferentes segmentos de rama como fuente de semilla vegetativa para siembra y la época apropiada para el corte de éstos. Se evaluaron 12 tratamientos (segmento apical, preapical, tercera y cuarta sección), con tres épocas de corte (60, 90 y 120 días después de siembra), en diseño de bloques completos al azar, en parcelas divididas, con tres repeticiones. El prendimiento de la semilla fue similar en todos los tratamientos, con promedio general de 98,73\%. Los tipos de segmentos afectan significativamente el rendimiento, siendo mejores el apical (11.643,96 kg ha-1) y preapical (11.101,47 $\left.\mathrm{kg} \mathrm{ha}^{-1}\right)$, que los otros. El menor rendimiento fue la cuarta sección (8.971,07 $\mathrm{kg} \mathrm{ha}^{-1}$ ). La época de corte de secciones no afectó los rendimientos. Sin embargo, época de corte 60 días y la sección apical son los de mayor rendimiento. Económicamente el mejor tratamiento fue la combinación sección apical con corte de 60 días.
\end{abstract}

Key words: Esquejes, prendimiento, rendimiento, rentabilidad.

1.- Corporación Colombiana de Investigación Agropecuaria (AGROSAVIA). Sede El Carmen de Bolívar - Km 1 vía Carmen de Bolívar-Zambrano, Teléfono +57(1)4227300 Ext 2294. El Carmen de Bolívar-Bolívar, Colombia. Código ORCID:0000-0002-7251-1374. Autor para correspondencia: rtamara@agrosavia.co

2.- Corporación Colombiana de Investigación Agropecuaria (AGROSAVIA). Centro de investigación Turipaná. Km 13 vía Montería - Cereté, Colombia. Teléfono +57(1)4227300 Ext. 2255. Cerete, Córdoba, Colombia. Código ORCID García-Peña, J.: 0000000218059487; Código ORCID Espitia-Montes, A.: 0000000280579483 Código ORCID Regino-Hernandez, S 0000000293257336; Código ORCID Perez-Cantero, S.: 0000-00015260-0321; Código ORCID Contreras-Santos, J.: 000000281793430.

Manuscrito recibido el 9 de octubre de 2019 y aceptado para su publicación el 2 de diciembre de 2020.

Támara Morelos, R. E; García Peña, J. A.; Espitia Montes, A. A.; Regino Hernández, S. M.; Pérez Cantero, S. P.; Contreras Santos, J. L. Evaluación de tipos de semilla y época de corte para la siembra de batata, Ipomoea batatas (L.) Lam, en la región Caribe de Colombia. FAVE - Ciencias Agrarias 19 (1): 111-120. CC BY-NC-SA 4.0 c(1) (2) 


\begin{abstract}
Evaluation of seed types and cutting season for the sowing of sweet potato, Ipomoea batatas (I) lam, in the Xaribbean region of Colombia

The objective was to evaluate different branch segments as a source of vegetative seed for sowing and the appropriate time for cutting these. Twelve treatments were evaluated. (apical, preapical, third and fourth section segment), with three cutting times (60, 90 and 120 days after planting), in a randomized complete block design, in divided plots, with three repetitions. Seed yield was similar in all treatments, with a general average of $98.73 \%$. The types of segments significantly affect performance, the apical $\left(11,643.96 \mathrm{~kg} \mathrm{ha}^{-1}\right)$ and preapical $\left(11,101.47 \mathrm{~kg} \mathrm{ha}^{-1}\right)$ being better than the others. The lowest yield was the fourth section $\left(8,971.07 \mathrm{~kg} \mathrm{ha}^{-1}\right)$. The cutting time of sections did not affect the yields. However, 60 day cutting time and the apical section are the ones with the highest performance. Economically the best treatment was the apical section combination with a 60-day cut.
\end{abstract}

Key words: Cuttings, sprout, performance, profitability.

\section{INTRODUCCIÓN}

La batata o boniato, Ipomoea batata (L.) Lam, es una especie cuyo origen se ubica en América, en donde posiblemente evolucionó independientemente en América Central (incluyendo el Caribe) y América del Sur (Venezuela, Colombia, Ecuador y Perú) (15; 13). Después del descubrimiento de América, esta especie se distribuyó en Europa, Asia y Oceanía y, dada la importancia que ha adquirido como fuente de carbohidratos, es un alimento saludable, casi perfecto. Y en los últimos tiempos su importancia radica más que nada en ser fuente de carotenos precursores de Vitamina A, sobre todo en países de Africa, donde tienen altos índices de ceguera. Hoy en día se cultiva comercialmente en aproximadamente 100 países en el mundo (9). La batata es una fuente importante de alimentos para gran parte de la población mundial (11), localizada principalmente en países tropicales (7); Se cultiva en más de 100 países con una producción cercana a 104.453.966 t/año en 8.029.864 ha plantadas en el mundo, ubicándose en el quinto puesto en importancia después del arroz, trigo, maíz y la mandioca (7). La batata es el tercero de los cultivos de raíces/tubérculos en importancia económica después de la papa y la mandioca (7). Asia es el continente de mayor producción y, entre los principales países productores, se destaca especialmente China, que genera cerca del $80 \%$ de la producción mundial. En América Latina se destacan México, Brasil, Argentina, Perú, Haití y Cuba, como los principales países productores. El rendimiento promedio mundial, del cultivo de batata se ubica en 12,60 t ha-1 (9). La batata es considerada el quinto alimento más importante para los países en desarrollo debido a sus características nutricionales, como alto aporte de vitamina A, proteínas, fibras, betacarotenos, precursores de vitamina A, azúcares, altas cantidades de almidón, otras vitaminas (B, E, etc), fibras (celulosa y pectinas) y minerales, entre los que se desta- 
ca el potasio $(9 ; 18)$. El sabor dulce de la batata se le atribuye a su alto contenido de sacarosa, glucosa y fructosa (14).

Aun cuando Colombia no figura en las estadísticas mundiales como un gran productor de esta raíz, su importancia desde el punto de vista de la seguridad alimentaria es relevante para algunas regiones del país (9). En la Región Caribe de Colombia, históricamente la batata se ha sembrado en forma tradicional en pequeñas áreas de terreno y parcelas, con materiales tipo criollo, utilizando semillas procedentes de raíces de baja calidad, esquejes de cualquier parte de la planta con edades superiores a las recomendadas, lo que genera bajas densidades por área sembrada y problemas fitosanitarios con plagas y enfermedades (9). La producción se destina principalmente para el autoconsumo de la población en las zonas rurales. Sin embargo, muy recientemente debido a la apertura de mercados en el exterior se ha despertado el interés por producir batata en forma tecnificada y comercial, destacándose los departamentos de Sucre, Magdalena y Córdoba, donde se concentran actualmente las mayores áreas de producción, resaltando al departamento de Sucre con el 53\% de la producción nacional (9).

Una de las mayores limitantes que enfrentan los productores en Colombia es la falta de semillas de buena calidad. Al respecto, se ha desarrollado muy poca investigación que permita determinar el tipo de semilla a utilizar y el momento oportuno de su obtención, de tal manera que se mejore el prendimiento en campo y así obtener mejores rendimientos. Aunque todo el tallo de la planta se puede usar como semilla, algunas investigaciones indican que la mejor fuente de semilla es la que se obtiene de sección apical de tallos maduros en plan- tas de 90 días de sembradas $(5 ; 8 ; 16)$. Los resultados de la investigación realizada por Birhanu et al. (2016) (3), evaluando el mejor tipo de corte para la variedad Awassa, sobre los parámetros de crecimiento, encontraron que para la brotación, número de hojas, diámetro de hojas, número de ramas primarias y secundarias, altura de la planta, volumen de raíces y circunferencia de la planta, los mejores resultados se obtuvieron con el tipo de corte de tallo apical, en comparación con el corte de la tercera sección del tallo.

La presente investigación se llevó a cabo con el propósito de determinar la mejor época para el corte de la semilla (esqueje) y el tipo de sección a utilizar para el establecimiento de cultivos de batata, en la Región Caribe Colombiana.

\section{MATERIALES Y MÉTODOS}

Localización: El presente estudio se llevó a cabo en la Corporación Colombiana de Investigación Agropecuaria (Agrosavia), Sede El Carmen de Bolívar, ubicada geográficamente a 942' 50.29” de latitud Norte y 7506' 27.2” de longitud Oeste, en el municipio del Carmen de Bolívar, departamento de Bolívar. Esta estación experimental se caracteriza por presentar una precipitación anual promedio de 1.100 mm, humedad relativa del $76 \%$, temperatura promedio anual de $27,7^{\circ} \mathrm{C}$. Pertenece a la formación de bosque seco tropical (BS-T) según la clasificación de Holdridge (2000) (10). El experimento se desarrolló bajo condiciones de campo, entre los meses de mayo de 2016 y enero de 2017. El suelo donde se instaló el ensayo presentó textura 
franco-arcillosa, $\mathrm{pH}=7,96 ; \mathrm{M} . \mathrm{O}=2,32 \%$; $\mathrm{P}=79,71 \mathrm{mg} \cdot \mathrm{kg}^{-1} ; \mathrm{Ca}^{2+}=28,71 \mathrm{Cmol} \cdot \mathrm{kg}^{-}$ ${ }^{1} ; \mathrm{Mg}^{2+}=3,07 \mathrm{mg} \cdot \mathrm{kg}^{-1} ; \mathrm{K}=0,63 \mathrm{mg} \cdot \mathrm{kg}^{-1}$; $\mathrm{CIC}=34,36 \mathrm{Cmol}_{\mathrm{kg}}{ }^{-1}$.

Material vegetal: Para estos experimentos se empleó la variedad de batata AGROSAVIA Aurora, estableciendo inicialmente semilleros bajo condiciones de vivero, para lo cual se usó raíz tuberosa. De estos semilleros se tomaron esquejes o segmentos de tallo de plantas totalmente formadas con edad de 60, 90 y 120 días. Para ello, se sembraron tres semilleros, el primero de los cuales se estableció el 18 mayo de 2016, el segundo: 30 días después (18 de junio de 2016) y el tercero, el 18 de julio de 2016

Para el presente artículo, la palabra semilla de batata, se refiere a los esquejes o segmentos de tallo que se obtiene de plantas bien desarrolladas (Plantas madre) y que son usados como material de propagación del cultivo, para siembra comercial. Los esquejes o segmentos tipo apical y preapical estuvieron entre 4 y $5 \mathrm{~mm}$ de grosos, en tanto que los tipo tercera y cuarta estuvieron entre 6 y $7 \mathrm{~mm}$. Todos los segmentos debían tener 4 nudos, para garantizar que 2 nudos queden dentro del suelo y los otros fuera, para emitir hojas y ramas.

Tratamientos: Se evaluaron 12 tratamientos consistentes en la combinación de cuatro segmentos o esquejes del tallo (apical, preapical, tercera y cuarta sección de guías o tallos), en combinación con tres edades de corte de los segmentos o esquejes (60, 90 y 120 días después de la siembra en vivero) (Tabla 1). De los semilleros se obtuvieron esquejes de $30 \mathrm{~cm}$ de longitud, los cuales fueron llevados a campo y sembrados, acorde a la distribución de los tratamientos (6).

Diseño experimental: Se utilizó un diseño experimental de bloques completos al azar, con arreglo en parcelas divididas, y tres repeticiones. La parcela principal correspondió a la época de corte de los esquejes (60, 90 y 120 días de edad de la planta madre al corte) y la subparcela, a la fuente de la semilla (apical, preapical, tercera y cuarta sección de las guías o tallos) (6).

Tabla 1. Tratamientos evaluados durante la investigación, Montes de María, Colombia 2017.

\begin{tabular}{|c|l|c|}
\hline Tratamiento & Segmento & Edad del corte (días) \\
\hline 1 & Apical & 60 \\
\hline 2 & Apical & 90 \\
\hline 3 & Apical & 120 \\
\hline 4 & Pre apical & 60 \\
\hline 5 & Pre apical & 90 \\
\hline 6 & Pre apical & 120 \\
\hline 7 & Tercera sección del tallo o guía & 60 \\
\hline 8 & Tercera sección del tallo o guía & 90 \\
\hline 9 & Tercera sección del tallo o guía & 120 \\
\hline 10 & Cuarta sección del tallo o guía & 60 \\
\hline 11 & Cuarta sección del tallo o guía & 90 \\
\hline 12 & Cuarta sección del tallo o guía & 120 \\
\hline
\end{tabular}


Unidad experimental: La unidad experimental estaba conformada por parcelas de cinco surcos distanciados a un metro y $5 \mathrm{~m}$ de largo, para un área de $25 \mathrm{~m}^{2}$ por parcela. El área efectiva total de la investigación fue de $300 \mathrm{~m}^{2}$. Los esquejes fueron sembrados a una distancia de un metro entre surcos $y$ $0,4 \mathrm{~m}$ entre plantas, para una población de 25.000 plantas ha $^{-1}$.

\section{Variables evaluadas}

Prendimiento: Se evaluó el prendimiento de los esquejes en campo a los ocho días después de la siembra, contando el número de esquejes vivos y prendidos en cada parcela. El porcentaje de prendimiento se calculó mediante la siguiente relación:

Prendimiento $(\%)=\frac{\text { No. esquejes vivos y prendidos } x 100}{\text { No. total de esquejes sembrados }}$

Rendimiento: A los 120 días después de la siembra se cosechó la totalidad de las raíces de batata producidas en los dos surcos centrales de cada parcela. Las raíces cosechadas se clasificaron acorde a su tamaño, forma y daños presentados, estableciendo dos categorías: primera categoría, correspondiente a raíces grandes, con un peso entre 80-1200 g, sin daños ni deformaciones; segunda categoría, correspondiente a raíces muy pequeñas, con un peso menor a $80 \mathrm{~g}$, raíces muy grandes, con un peso superior a 1200 g y raíces con daños y deformaciones. El rendimiento se calculó, para ambas categorías, mediante la siguiente relación:

Rendimiento $\left(\mathrm{kgha}^{-1}\right)=\frac{\text { Peso de la parcela }(\mathrm{g}) \times 10}{\text { Area de la parcela }(\mathrm{m} 2)}$
Análisis económico: Se llevó un registro detallado de los costos de producción para cada tratamiento, los rendimientos obtenidos y el precio de venta de la batata. El precio de venta en su conjunto, tanto de raíces de primera y segunda categoría fue de \$250.000 por tonelada. Con lo anterior se estimaron los ingresos brutos y netos, para luego estimar la rentabilidad para cada tratamiento, empleando la ecuación:

$$
\text { Re } n t=\frac{I B-C T}{C T} * 100
$$

Donde IB es el ingreso bruto $\left(\$ . h a^{-1}\right)$ y CT es el costo total $\left(\$ \cdot h a^{-1}\right)$. Los costos se obtuvieron mediante el registro de los costos de producción del cultivo.

Análisis de datos: Se realizaron análisis de varianza para cada variable y en los casos en los cuales se detectaron diferencias a un nivel de significancia del 0,05 , se realizaron pruebas de separación de medias utilizando la HSD de Tukey (Honestly-Significant-Difference). Para lo anterior se utilizó el paquete estadístico SAS versión 9.4 (SAS Institute, 2008).

\section{RESULTADOS Y DISCUSIÓN}

Prendimiento: El análisis de varianza realizado a esta variable no detectó diferencias significativas para la interacción entre las épocas de corte y los tipos de semilla evaluados. Tampoco se encontraron diferencias para los efectos principales de épocas de corte y tipos de semilla. Estos resultados indican que la época del corte y el tipo de semilla no afectan los porcentajes de prendimiento de los esquejes en campo. En promedio, las tres épocas de corte de la 
semilla y los cuatro tipos de semilla proveyeron similares prendimientos en campo, con un promedio general de $98,73 \%$ (figura 1) (6) con coeficiente de variación de 2,37\%. Estos porcentajes de prendimiento se consideran aceptables desde el punto de vista de las siembras comerciales de batata. Investigaciones realizadas por otros autores indican que la mejor época para tomar semilla para siembra de batata es la comprendida entre los 60 y 90 días después de la siembra (12). Estos resultados se asemejan a los encontrados en la presente investigación (figura 1), observando el menor prendimiento en la sección cuarta del tallo, debido a que, al envejecer los esquejes, se reduce la capacidad de enraizamiento (1). Birhanu et al. (2016) (3), encontraron que el mayor prendimiento de los esquejes se presentó cuando utilizaron la sección apical del tallo.
Rendimientos: Con relación a los rendimientos por categoría, el análisis de varianza mostró que el rendimiento de primera no fue afectado por la época de corte, el tipo de semilla ni por la interacción entre los dos, dado que no se encontró diferencia estadística. En cuanto al rendimiento de segunda, se presentó interacción $(\mathrm{p}<0.05)$ y la combinación época de corte de 60 días por el tipo de semilla apical mostró los mejores resultados con 6.367,52 kg.ha-1. El menor rendimiento fue la combinación de 120 días por semilla de la sección cuarta del tallo, con 1.193,83 kg.ha-1. Este rendimiento mostró un comportamiento similar a la tendencia mostrada por el rendimiento total, corroborándose que la época de corte 60 días y la sección apical son los de mayores registros de este atributo en batata.

\section{del tallo,}
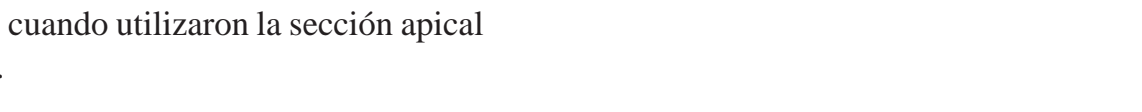

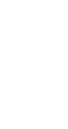


Para el rendimiento total, no se detectaron raíces de primera categoría fue siempre madiferencias significativas en la interacción yor que la de segunda, excepto en la época época de corte y tipo de semilla, pero sí de corte de 60 días y el tipo de semilla apiexistieron $(p<0,05)$ entre los tipos de se- cal, en que no hubo diferencias entre ambas milla, obteniendo mayores rendimientos de categorías (Tabla 2).

campo con la utilización de esquejes de la parte apical de los tallos o guías (6). El tratamiento con los menores rendimientos de campo fue el correspondiente a la siembra de esquejes obtenidos de la sección cuarta de los tallos o guías obtenida a los 120 días

De lo anterior se puede concluir que, para el establecimiento de los cultivos en campo, se puede obtener igual prendimiento de los esquejes, independientemente del tipo y edad de la semilla utilizada. Sin embargo, los mejores efectos productivos se de sembrada la planta. La producción de obtienen con los esquejes obtenidos de la

Tabla 2: Rendimiento total $\left(\mathrm{kgha}^{-1}\right)$ de raices de batata variedad Agrosavia Aurora de pulpa naranja, por efecto de la edad y tipo de esqueje como material de siembra en Montes de María, Colombia 2017.

\begin{tabular}{|c|c|c|c|c|}
\hline $\begin{array}{l}\text { Época de corte } \\
\text { (días) }\end{array}$ & $\begin{array}{l}\text { Tipo de } \\
\text { semilla }\end{array}$ & $\begin{array}{l}\text { Rendimiento primera } \\
\text { categoría }\left(\mathrm{kgha}^{-1}\right)^{1}\end{array}$ & $\begin{array}{c}\text { Rendimiento } \\
\text { segunda categoría } \\
\left(\mathrm{kgha}^{-1}\right)^{1}\end{array}$ & $\begin{array}{l}\text { Rendimiento total } \\
\qquad\left(\mathrm{kgha}^{-1}\right)^{1}\end{array}$ \\
\hline 60 & Apical & $6.180,00 \pm 1744,12$ & $6.367,81 \pm 877,61$ & $12.547,81 \pm 1.629,88$ \\
\hline 60 & $\begin{array}{l}\text { Pre } \\
\text { Apical }\end{array}$ & $6.762,10 \pm 2343,99$ & $4.713,68 \pm 2162,65$ & $11.475,78 \pm 2.824,84$ \\
\hline 60 & $\begin{array}{l}\text { Tercera } \\
\text { sección }\end{array}$ & $6.438,10 \pm 3024,35$ & $4.274,51 \pm 980,62$ & $10.712,61 \pm 3.238,43$ \\
\hline 60 & $\begin{array}{l}\text { Cuarta } \\
\text { Sección }\end{array}$ & $5.924,55 \pm 648,50$ & $3.430,00 \pm 380,66$ & $9.354,55 \pm 499,67$ \\
\hline 90 & Apical & $6.482,65 \pm 1226,95$ & $5.363,24 \pm 379,91$ & $11.845,89 \pm 1.127,08$ \\
\hline 90 & $\begin{array}{l}\text { Pre } \\
\text { Apical }\end{array}$ & $7.532,32 \pm 744,18$ & $3.499,22 \pm 801,60$ & $11.031,54 \pm 1.347,04$ \\
\hline 90 & $\begin{array}{l}\text { Tercera } \\
\text { sección }\end{array}$ & $5.736,94 \pm 1327,37$ & $4.540,70 \pm 2645,48$ & $10.277,64 \pm 2.656,92$ \\
\hline 90 & $\begin{array}{l}\text { Cuarta } \\
\text { Sección }\end{array}$ & $6.212,60 \pm 3771,31$ & $3.287,21 \pm 1098,97$ & $9.499,81 \pm 4.604,94$ \\
\hline 120 & Apical & $7.337,10 \pm 2005,70$ & $3.201,08 \pm 739,33$ & $10.538,18 \pm 2.305,41$ \\
\hline 120 & $\begin{array}{l}\text { Pre } \\
\text { Apical }\end{array}$ & $6.163,08 \pm 969,96$ & $4.634,00 \pm 2592,94$ & $10.797,08 \pm 2.465,04$ \\
\hline 120 & $\begin{array}{l}\text { Tercera } \\
\text { sección }\end{array}$ & $7.025,14 \pm 1426,03$ & $2.588,94 \pm 1428,28$ & $9.614,08 \pm 1.039,04$ \\
\hline 120 & $\begin{array}{l}\text { Cuarta } \\
\text { Sección }\end{array}$ & $6.865,05 \pm 951,30$ & $1.193,80 \pm 358,39$ & $8.058,85 \pm 916,62$ \\
\hline Media general & & $6.554,97$ & $3.924,52$ & $10.479,49$ \\
\hline R square & & 0,10 & 0,50 & 0,27 \\
\hline CV\% & & 29,23 & 36,77 & 22,38 \\
\hline $\begin{array}{l}\text { Significancia } \\
\text { Época }\end{array}$ & & ns & * & ns \\
\hline $\begin{array}{l}\text { Significancia } \\
\text { Tipo }\end{array}$ & & ns & * & * \\
\hline $\begin{array}{l}\text { Significancia } \\
\text { Epoca x Tipo }\end{array}$ & & ns & * & ns \\
\hline
\end{tabular}

(1) Promedio de tres repeticiones; $n s$ : no significativo ( $p \geq 0.05)$; *: significativo $(p<0.05)$ 
parte apical de las guías o tallos (6). Estos resultados concuerdan con los obtenidos por diferentes autores, quienes indican que los esquejes obtenidos de la parte apical de los tallos dan lugar a mejores rendimientos, esto se debe a que, en el segmento apical del tallo, se encuentra el punto de crecimiento, lo que permite mejor prendimiento y desarrollo de la planta, resultando al final un mayor rendimiento $(5 ; 17 ; 2 ; 4)$. Por su parte Sulbarán (2008) (16) encontró que, utilizando propagación por medio de esquejes de la sección apical, se obtuvo el mayor peso de la parte aérea de la planta.

Esquejes apicales tienen menos probabilidad de portar patógenos. Por su parte, Birhanu et al, (2016) (3), reportan que el uso de esquejes apícales favorece el desarrollo de las raíces, lo cual también podría tener influencias sobre los rendimientos.

Análisis económico. El análisis económico tuvo en cuenta la rentabilidad de los tratamientos (tabla 3), encontrando que el mejor promedio lo obtuvo la época de corte realizada a los 60 días con la sección apical del tallo (7,9\%) y la más baja la presentó la época de corte a los 120 días y el tipo de semilla del cuarto segmento (-27,1\%). Este resultado obedece a que en la combinación del corte a los 60 días con la sección apical presentaron los mayores rendimientos $\left(12.547,81 \mathrm{~kg} \cdot \mathrm{ha}^{-1}\right)$, el menor rendimiento lo obtuvo el corte a los 120 días con la sección cuarta del tallo (8.058,85 kg.ha-1) (6). Los resultados, para los demás tratamientos, estuvieron comprendidos entre estos dos valores de rentabilidad (Tabla 3).

La presente tecnología muestra eficiencia desde el punto de vista económico al disminuir el costo unitario del producto de \$342.908 (USD 101,33) (cuando se usa como material de siembra el cuarto segmento cortado a los 120 días) a \$231.717 (USD 68,47) (cuando se emplea el segmento apical cortado a los 60 días), significando una reducción del $47 \%$ en los costos para la región Montes de María (tabla 3). Los resultados del análisis económico estuvieron basados en los costos de producción,

Tabla 3. Análisis económico comparativo de costos de producción de batata variedad Agrosavia Aurora utilizando tipos de segmentos de semilla (esquejes) y épocas de corte en el cultivo de batata, Montes de María, Colombia 2017.

\begin{tabular}{|l|l|l|l|l|l|l|c|}
\hline $\begin{array}{c}\text { Época de } \\
\text { corte (días) }\end{array}$ & \multicolumn{1}{|c|}{$\begin{array}{c}\text { Tipo de } \\
\text { semilla }\end{array}$} & $\begin{array}{c}\text { Costos } \\
\text { Total } \\
\left(\$ \mathbf{h a}^{-1}\right)\end{array}$ & $\begin{array}{c}\text { Costos } \\
\text { Unitario } \\
(\$ / \mathrm{t})\end{array}$ & $\begin{array}{c}\text { Rend. } \\
\left(\text { (t.ha }^{-1}\right)\end{array}$ & $\begin{array}{l}\text { Ingreso } \\
\text { Bruto }(\$)\end{array}$ & $\begin{array}{l}\text { Ingreso } \\
\text { Neto }(\$)\end{array}$ & $\begin{array}{c}\text { Rentabilidad } \\
(\%)\end{array}$ \\
\hline 60 & Apical & 859,20 & 68,47 & 12,55 & 927,00 & 67,79 & 7,9 \\
\hline 60 & Preapical & 849,03 & 73,98 & 11,48 & 847,80 & $-1,24$ & $-0,1$ \\
\hline 60 & Tercera & 841,79 & 78,58 & 10,71 & 791,42 & $-50,38$ & $-6,0$ \\
\hline 60 & Cuarta & 828,91 & 88,61 & 9,35 & 691,09 & $-137,82$ & $-16,6$ \\
\hline 90 & Apical & 852,54 & 71,97 & 11,85 & 875,14 & 22,60 & 2,7 \\
\hline 90 & Preapical & 844,82 & 76,58 & 11,03 & 814,98 & $-29,84$ & $-3,5$ \\
\hline 90 & Tercera & 837,67 & 81,50 & 10,28 & 759,28 & $-78,38$ & $-9,4$ \\
\hline 90 & Cuarta & 830,29 & 87,40 & 9,50 & 701,82 & $-128,47$ & $-15,5$ \\
\hline 120 & Apical & 842,59 & 78,04 & 10,80 & 797,66 & $-44,94$ & $-5,3$ \\
\hline 120 & Preapical & 842,59 & 78,04 & 10,80 & 797,78 & $-44,94$ & $-5,3$ \\
\hline 120 & Tercera & 831,37 & 86,47 & 9,61 & 710,26 & $-121,11$ & $-14,6$ \\
\hline 120 & Cuarta & 816,62 & 101,33 & 8,06 & 595,36 & $-221,26$ & $-27,1$ \\
\hline
\end{tabular}

Nota: valor de la tasa de cambio de dólar a pesos colombianos es de 3.384 pesor por dollar. 
rendimiento y precio de venta del producto que, para este caso, fue de $\$ 250.000$ (USD $73,88)$ por tonelada de batata.

\section{CONCLUSIONES}

El desarrollo de la investigación permitió establecer que la combinación entre época de corte y tipo de semilla no influyen o inciden en el prendimiento de los esquejes de batata, por tanto, cualquier tipo de semilla con la madurez fisiológica suficiente se puede usar en la región de los Montes de María.

Sólo el tipo de semilla influye significativamente en el rendimiento, siendo el mejor el segmento apical con rendimiento promedio de 11.643,96 $\mathrm{kgha}^{-1}$, seguido del preapical 11.101,47 kg.ha ${ }^{-1} \mathrm{y}$ del tercero con un rendimiento promedio de $10.201,44$ kg.ha-1 .

La época de corte de la semilla no influye en el rendimiento, por tanto este parámetro es indiferente para la producción de material de siembra.

Económicamente, la mejor semilla se obtiene con la combinación de segmento apical, cortada a los 60 días después de siembra; donde el retorno es de 7,9\% de rentabilidad neta.

\section{AGRADECIMIENTOS}

La presente investigación fue financiada por el Ministerio de Agricultura y Desarrollo Rural (MADR) de Colombia, con el convenio TV-16, mediante el cual se ejecutó el proyecto: "Validación y generación de tecnologías para el manejo del cultivo de batata”. Agradecimientos al equipo de investigación del Centro de Investigación Turipaná y la Sede Carmen de Bolívar de la Corporación Colombiana de Investigación Agropecuaria (AGROSAVIA) por su apoyo científico, técnico y logístico en la ejecución de la investigación. Agradecimientos a CLAYUCA por ser la fuente del material reproductivo con el cual se investigó.

\section{BIBLIOGRAFIA}

1.- AHMED, I.; HASSAN A.; ABDUR R. AND N. ALI. 2000. Yield dynamic in potato relation to variety and row spacing.Pak.J. Biol. Sci.3:1247-1249.

2.- ALBAN, A. 2005. CLAYUCA, Consorcio latinoamericano de apoyo a la investigación y desarrollo de la yuca y batata. Producción de semillas de batata en invernaderos.

3.- BIRHANU L.; ADANECH, B. \& GENET D. 2016. The Evaluation of Growth Performance of Sweet Potato (Ipomoea Batatas L.) Awassa Var. by Using Different Type of Vine Cuttings. Wolaita Sodo University, College of Agriculture, Department of Horticulture. Artículo en: Rev. Food Science and Quality Management, www.iiste.org ISSN 22246088 (Paper) ISSN 2225-0557 (Online) Vol.54, 2016.

4.- CADAVID, L; ALBAN, A. 2004. Yuca y batata una alianza de grandes proyecciones. Boletín electrónico de CLAYUCA .http:// www. Clayuca .org/ clayucanet / noticias batata.htm.

5.- CAÑAS, K.; GONZÁLEZ, V. \& MARTÍNEZ, R. 2016. Evaluación de tres tipos de esquejes de la guía principal (apical, intermedia y basal) de tres variedades de camote (Ipomoea batatas L.) con la finalidad de determinar la mejor producción. Tesis de pre- 
grado. Facultad de Agronomía, Universidad de El salvador, El Salvador. http://ri.ues.edu. sv/id/eprint/11929/1/13101618.pdf. Recuperado 28/05/2019.

6.- CORPORACIÓN COLOMBIANA DE INVESTIGACIÓN AGROPECUARIA “AGROSAVIA". 2017. Informe final Macroproyecto: Desarrollo y vinculación de tecnologías para sistemas de producción de ñame y batata para consumo fresco, transformación y exportación en el Caribe Colombiano, Proyecto: Validación y generación de tecnologías para el manejo del cultivo de batata. Centro de Investigación Turipaná, Cereté y Carmen de Bolívar.

7.- FAOSTAT. Anuario Estadístico de la FAO. 2016. (En línea). (Consultado: febrero de 2019). Disponible en: faostat.fao.org/

8.- FIGUEROA, S. 2015. Evaluación de dos sistemas de producción de semilla prebásica de camote (Ipomoea batatas L.). Tesis de pregrado. Facultad de Agronomía. Departamento Académico de Fitotecnia. Universidad Nacional Agraria La Molina. Lima, Perú.

9.- FLÓREZ, D.; CONTRERAS, C. y URIBE, C. 2016. Perspectivas tecnológicas y comerciales para el cultivo de la batata en Colombia. Mosquera (Colombia): Corporación Colombiana de Investigación Agropecuaria (Corpoica). 110 p. ISBN (e): 978-958-740223-0.

10.- HOLDRIDGE, L.R. 2000. Ecología basada en zonas de vida. Quinta reimpresión. San José (Costa Rica): Instituto Interamericano de Cooperación para la Agricultura (IICA). p. 216.San José, CRCI.

11.- KOODI, S.; SINGH, S. P.; ROLANIYA, M. K. \& RAJ, P. 2017. The Growth, yield and quality of sweet potato (Ipomoea batatas Lam.) Influenced by different plant densities. IJCS, 5(4), 359-361.
12.- PUENTE, M.; GARCÍA J.; MARTÍ, H.; PERTICARI, A. \& ULLÉ, J. 2015. Respuesta de dos variedades de batata frente a la inoculación con microorganismos promotores del crecimiento vegetal según el tipo de estaca. P 1-6. En: Memorias del V Congreso Latinoamericano de Agroecología. Instituto de Microbiología y Zoología Agrícola, INTA; Argentina; EEA INTA San Pedro, Argentina. Archivo Digital: descarga y online ISBN 978-950-34-1265-7.

13.- LOEBENSTEIN, G. \& THOTTAPPILLY, G. 2009. The Sweetpotato. Washington D.C., EE. UU.: Springer.

14.- MARTÍ, H.R.; D’CHUDIL, H. \& CORBINO, G. 2011. La batata, el redescubrimiento de un cultivo. Cienc Hoy. 21(121):17-23.

15.- MORALES, A.; MORALES, A.; RODRÍGUEZ, D.; PASTRANA, I. \& MÉNDEZ, C. 2017. Origen, evolución y distribución del boniato (Ipomoea batatas (L.) Lam.). Una revisión. Rev. Agricultura Tropical Vol. 3 No. 1:1-13, 2017 ISSN on line: 2517-9292.

16.- SULVARAN, L.; GONZÁLEZ, C.; ARAQUE, H.; VERDE, O. \& LAPP, A. 2008. Efecto de dos tipos de propagación asexual sobre el rendimiento de dos cultivares de batata (Ipomoea batatas L.). Rev. Unell. Cienc. Tec. 26: 6-13.

17.- RODRÍGUEZ, G. 2008. Caracterización de variedades de batata (ipomonea batata) con el fin de desarrollar un puré que sea fuente para la elaboración de productos preformados en McCain Colombia. Tesis de pregrado. Ingeniería de Alimento, Universidad de la Salle, Bogotá, Colombia. P, 9.

18.- VARGAS, P. \& HERNÁNDEZ, D. 2013. Harinas y almidones de yuca, ñame, camote y ñampí: propiedades funcionales y posibles aplicaciones en la industria alimentaria. Revista Tecnología en Marcha, 26(1), Pág-37. 